\title{
Factores que tienen impacto en la generación de valor de una oficina de gestión de proyectos de TIC, en organizaciones grandes del Estado de Nuevo León (Factors that have an impact on theue generation of an ICT project management office in large organizations of the State of Nuevo León)
}

\author{
Jesús Garza Sánchez \\ Miguel Ángel Palomo González*
}

\begin{abstract}
It is common to see in organizations that are continually seeking administrative efficiencies that result in cost reduction, especially in times with economic pressures, even in those that can be financially healthy, reduce fixed costs through layoffs, regularly in departments that apparently do not generate income. Information and communications technology project management offices have not been fully accepted as an indispensable part in modern organizations; they must justify their existence by demonstrating that they can improve the performance of a company by modernizing or restructuring operations; they often have difficulty demonstrating their value to other executives; this is a serious problem, so that about half disappear after 2 years of having started; The above can happen even when they have a positive effect on the company; because in many cases, they do not show their benefit correctly (O'Brochta, M 2002). The foregoing also applies to Mexican organizations. However, these offices have arguments with which they can justify their existence. On the other hand; there is a continuing concern of ICT PMOs to measure their own performance, according to the study carried out by doctors Hobbs \& Aubry (2007, p. 82) where they conclude that $50 \%$ of the cases studied had the function of monitoring and control their own performance.
\end{abstract}

Key words: information and communications technology, international Organization for Standarization, Project Management Book of Knolwedge, Project Management Institute, project management office

JEL: C39, O32.

\footnotetext{
- Universidad Autónoma de Nuevo León, Facultad de Contaduría Pública y Administración, Centro de Desarrollo Empresarial y Posgrado, Av. Universidad S/N, San Nicolás de los Garza , N.L., México, C.P. 66455, (+52) 8113 4044 30, jesgarza@hotmail.com

* Universidad Autónoma de Nuevo León, Facultad de Contaduría Pública y Administración, Centro de Desarrollo Empresarial y Posgrado, Av. Universidad S/N, San Nicolás de los Garza, N.L., México, C.P. 66455, (+52) 8113 4044 30, mapalomo2003@yahoo.com
} 
Resumen. Es común ver en las organizaciones que continuamente están buscando eficiencias administrativas que traigan como resultado la reducción de costos, sobre todo en épocas con presiones económicas, aún en aquellas que pueden ser financieramente saludables, realizan disminución de gastos fijos por medio de despidos, regularmente en departamentos que aparentemente no generan ingresos. Las oficinas de administración de proyectos de tecnología de información y comunicaciones no han sido totalmente aceptadas como parte indispensable en las organizaciones modernas; deben justificar por si mismas su existencia demostrando que pueden mejorar el desempeño de una compañía al modernizar o reestructurar las operaciones; a menudo tienen dificultad para demostrar su valor a otros ejecutivos; esto les constituye un serio problema, de tal forma que cerca de la mitad desaparecen después de 2 años de haber iniciado; lo anterior puede ocurrir aún y cuando tengan efecto positivo en la compañía; debido a que en muchos de los casos, no evidencian correctamente su beneficio (O'Brochta, M 2002). Lo anterior también aplica para las organizaciones Mexicanas. Sin embargo, estas oficinas disponen de argumentos con los cuáles pueden justificar su existencia. Por otra parte; existe una continua preocupación de estas oficinas por medir su propia actuación, lo anterior de acuerdo al estudio realizado por los doctores Hobbs \& Aubry (2007, p. 82) en donde concluyen que el $50 \%$ de los casos estudiados tenían como función monitorear y controlar su propia actuación.

Palabras clave: Instituto de Gestión de Proyectos, oficina de administración de proyectos, Organización Internacional de Normalización, PMBOK, tecnología de información y comunicaciones.

\section{Introducción}

La Guía del Project Management Body of Knowledge (PMBOK® Guide) define una Project Management Office (PMO Oficina de administración de proyectos) como: "Una entidad organizacional a la que se le asignan varias responsabilidades relacionadas con la administración centralizada y coordinada de aquellos proyectos a su cargo. Como se menciona en el PMI, PMBOK Guide (2012, p. 369), el rango de las responsabilidades puede ser desde proporcionar funciones de soporte a la administración de proyectos hasta hacerse responsable de manera directa de la administración de un proyecto, pueden operar en aspectos que van desde proporcionar las funciones de respaldo para la dirección de proyectos bajo la forma de formación, software, políticas estandarizadas y procedimientos, hasta la dirección y responsabilidad directas en sí mismas para lograr los objetivos del proyecto. De acuerdo a Baker (2007); Dai \& Wells (2006); Hobbs \& Aubrey 
(2007); y Kwak \& Dai (2000) son unidades centralizadoras de proyectos dentro de las organizaciones que se focalizan en asegurar que terminen en tiempo, presupuesto y alcance.

\section{Marco teórico}

Antecedentes teóricos generales del tema

Desde finales de los 90 , se ha presentado un movimiento importante a nivel mundial tendiente a la creación de oficinas de administración de proyectos de TIC y se ha incrementado durante la primer década del 2000 (Dai \& Wells, 2004, p. 524; Hobbs \& Aubry, 2007, p.74). A pesar de la percepción común, de que un importante número de las principales organizaciones en el mercado tienen, al menos una oficina de este tipo en su estructura organizacional, Hurt \& Thomas (2009, p.55), mencionaron que "la sustentabilidad de las PMO TIC es un tema débil".

De hecho, surgen debido a diferentes necesidades, pero la gran mayoría tienen un objetivo en común: Obtener mejores resultados en aquellos proyectos desarrollados por la organización. (Pinto, De Matheus y Levin, 2010).

En los últimos años, mientras que algunas prosperaron y maduraron y crearon notorio valor para la organización, otras han perdido su fuerza y apoyo y han sufrido recortes y reducciones 0 han sido eliminadas por completo. Adicionalmente, Hobbs \& Aubry (2007, p. 74) en su investigación titulada A Multi-Phase Research Program Investigating Project Mangement Offices, vieron que había una variación significativa en la estructura, el rol asumido, y el valor percibido de las PMO TIC, demostrando con esto la inestabilidad y diversidad del rendimiento o actuación de dichas oficinas. En este estudio se muestra un retrato confiable de la población de estas oficinas, en donde se observa que el $50 \%$ de las estudiadas, tienen la actividad de monitorear y controlar su propia actuación. A continuación en la Tabla 1 se muestran los resultados mencionados: 
Tabla 1. Funciones Principales que realiza una PMO de TIC.

\begin{tabular}{lc}
\hline \multicolumn{1}{c}{ Función de la PMO TIC } & $\begin{array}{c}\% \text { PMOs en donde } \\
\text { es importante }\end{array}$ \\
\hline Reportar estatus de los proyectos a la alta administración & $83 \%$ \\
Desarrollar e implementar una metodología estándar & $76 \%$ \\
Monitorear y controlar el desempeño de los proyectos & $65 \%$ \\
Desarrollar competencias del personal, incluyendo entrenamiento & $65 \%$ \\
Implementar y operar un sistema de información de proyectos & $60 \%$ \\
Proporcionar asesoría a la alta administración & $60 \%$ \\
Coordinación inter proyectos & $59 \%$ \\
Desarrollar y mantener un project scoreboard & $58 \%$ \\
Promover la administración de proyectos dentro de la organización & $55 \%$ \\
Auto Monitoreo y control del performance de la PMO & $50 \%$ \\
Participar en la planeación estratégica & $49 \%$ \\
Proporcionar mentoría a los gerentes de proyecto & $49 \%$ \\
Administrar uno o más portafolios & $49 \%$ \\
Identificar, seleccionar, y priorizar nuevos proyectos & $48 \%$ \\
Administrar el almacenamiento de la documentación de proyectos & $48 \%$ \\
Administrar uno o más programas & $48 \%$ \\
Dirigir auditorías de proyectos & $45 \%$ \\
Administrar las interfaces con los clientes & $45 \%$ \\
Proporcionar un conjunto de herramientas personalizables para satisfacer & $42 \%$ \\
las necesidades de programas y proyectos & \\
Ejecutar tareas especializadas (servicios) para los gerentes de proyectos & $42 \%$ \\
Asignar (y compartir) recursos dentro de los proyectos & $40 \%$ \\
Dirigir revisiones post implementación (Lecciones Aprendidas) & $38 \%$ \\
Implementar y administrar bases de datos de lecciones aprendidas & $34 \%$ \\
Implementar y administrar bases de datos de riesgos & $29 \%$ \\
\hline Futa & \\
\hline
\end{tabular}

Fuente: A multiphase research program investigating project management offices. Hobbs, B. \& Aubry, M. (2007).

Las oficinas exitosas enfrentan el reto de asegurar que sus prácticas, continuamente maduren conforme la propia evolución de la organización y esto les demanda estar alineadas a las nuevas necesidades, resultado de esos cambios, logrando con que se incremente la percepción de valor que se tiene de ellas.

Al igual que cualquier una unidad organizacional generadora de servicios, son continuamente cuestionadas acerca de su contribución a la organización, y a menudo, son vistas como un tipo de sobrecarga operativa.

Se le considera a menudo como un área de soporte dentro de la organización, de manera similar a las áreas de Contabilidad, Recursos Humanos, Seguridad, Tecnología de Información y Comunicaciones, en la mayoría de las organizaciones, estas áreas no son consideradas como un 
área que tengan su propia razón de ser, ya que su objetivo es apoyar el desarrollo de la actividad principal de la compañía. Sin embargo, existen diferencias importantes que las hacen distintas de las otras áreas de apoyo. El requisito de que deben generar valor de negocio es, de hecho, algo mucho más crítico que el exigido normalmente en las áreas de apoyo tradicionales, valor que se traduce en beneficios que no siempre son perceptibles 0 medibles. (Pinto et al, 2010), deben justificar por si mismas su existencia demostrando que pueden mejorar el desempeño de una compañía. Desde otra perspectiva, pueden considerarse como un proveedor de servicios para la organización, su continuidad se vuelve frágil, si tenemos en mente que pueden perder todo el respaldo y apoyo que tienen, si no son capaces de aportar mejoras que se perciban y justifiquen su inversión.

\section{Variable dependiente (Generación de valor empresarial)}

Considerando el enfoque que se dio al estudio, se revisó el resultado de diferentes estudios e investigaciones realizadas acerca de la evolución y desempeño de las PMO TIC, por instituciones prestigiadas como el Instituto de Administración de Proyectos (PMI, por sus siglas en inglés).

Definir la generación de valor es difícil; depende de quién sea el observador, quién la perciba / reciba. De manera general podría decirse que significa recibir lo que se espera o que lo recibido supera las expectativas. El verdadero valor de estas oficinas radica en resaltar la relación crítica entre su trabajo y los resultados del negocio. (Ward, 2010, p. 25).

Lo anterior significa que pueden demostrar, por ejemplo, que con su actuación ayudan a reducir costos, mejorar la rentabilidad, obtener el retorno sobre la inversión (ROI, return on investment, por sus siglas en inglés) en los proyectos de TIC, mejora el número de proyectos exitosos, asegura que se satisfacen todos los requerimientos del cliente, se aumenta el nivel de satisfacción de los clientes, se incrementan: La productividad, ventas, ingresos, ganancias, participación de mercado.

Además se puede decir que el valor de negocio de proyectos de TIC se genera cuando se seleccionan los proyectos adecuados para la organización, se gestionan de manera profesional y se ejecutan cumpliendo 0 superando las expectativas del cliente. Algunos de los autores que han estudiado el tema para la generación de valor empresarial de una PMO TIC 
son: Hurt \& Thomas (2009), Stanleigh (2008, 2009),Young \& Xiao Yi Dai (2000), Andersen, Bjolrn1, Henriksen, Bjolrnar2, Aarseth, Wenche2 (2007), Duggal (2006, 2009, 2010a, b), Ward (2010), Brantley (2007), PMBOK (2012), Barba (2010), Pinto, De Matheus, y Levin (2010), PMI (2014).

\section{Tabla 2. Matriz teórica de variables independientes y dependiente}

\begin{tabular}{|c|c|c|}
\hline Variables & Definición conceptual & Autores \\
\hline $\begin{array}{l}\text { X1 Alineación a } \\
\text { Objetivos } \\
\text { Estratégicos de la } \\
\text { Organización }\end{array}$ & $\begin{array}{l}\text { Asegurar que todos los proyectos a realizar estén } \\
\text { alineados con la Planeación Estratégica de la } \\
\text { Organización. }\end{array}$ & $\begin{array}{l}\text { (Hurt \& Thomas, 2009), } \\
\text { (Stanleigh, 2008), (Young H.\& } \\
\text { Xiao Yi Dai, 2000), (Project } \\
\text { Management Institute, Inc. } \\
\text { PMl.org/Pulse, 2013, 2014) }\end{array}$ \\
\hline $\begin{array}{l}\text { X2 Tiempo } \\
\text { Acordado }\end{array}$ & $\begin{array}{l}\text { Administrar el número de recursos y sus cargas de } \\
\text { trabajo así como fechas reales / alcanzables. }\end{array}$ & $\begin{array}{l}\text { (Stanleigh, 2008), (Andersen, } \\
\text { Bjolrn1, Henriksen, Bjolrnar2, } \\
\text { Aarseth, y Wenche2, 2007), } \\
\text { (Duggal, 2010a). }\end{array}$ \\
\hline $\begin{array}{l}\text { X3 Presupuesto } \\
\text { Acordado }\end{array}$ & $\begin{array}{l}\text { Administrar adecuadamente los costos de los } \\
\text { proyectos. }\end{array}$ & $\begin{array}{l}\text { (Hurt \& Thomas, 2009), } \\
\text { (Stanleigh, 2008). }\end{array}$ \\
\hline $\begin{array}{l}\text { X4 Alcance } \\
\text { Acordado }\end{array}$ & $\begin{array}{l}\text { Declarar con claridad los entregables así como lo } \\
\text { que no se incluirá. Nivel de cumplimiento de las } \\
\text { especificaciones iniciales. Se puede hablar de un } \\
\text { alcance Organizacional, Geográfico, Operativo, } \\
\text { Funcional. }\end{array}$ & $\begin{array}{l}\text { (Hurt \& Thomas, 2009), } \\
\text { (Stanleigh, 2009), (Duggal, } \\
\text { 2010a) }\end{array}$ \\
\hline $\begin{array}{l}\text { X5 Calidad } \\
\text { Acordada }\end{array}$ & Los proyectos terminados deben tener cero errores & $\begin{array}{l}\text { (Hurt \& Thomas, 2009), } \\
\text { (Stanleigh, 2009), (Duggal, } \\
\text { 2010a) }\end{array}$ \\
\hline $\begin{array}{l}\text { X6 Lograr la } \\
\text { Satisfacción de los } \\
\text { Clientes }\end{array}$ & $\begin{array}{l}\text { Un estado de cumplimiento en el cual las } \\
\text { necesidades de los clientes se satisfacen o se } \\
\text { superan respecto a las expectativas del cliente } \\
\text { según las considere al momento de la evaluación. } \\
\text { Los proyectos terminados cumplen con la totalidad } \\
\text { de los requerimientos / necesidades de sus } \\
\text { clientes. Incluye dos elementos: 1) Conformidad } \\
\text { con los requisitos: El proyecto produce lo que dijo } \\
\text { que produciría, aquello para lo cual fue } \\
\text { emprendido. 2) Adecuado para el uso / Fitness for } \\
\text { use: El producto o servicio satisface necesidades } \\
\text { reales. }\end{array}$ & $\begin{array}{l}\text { (Stanleigh, 2009), (Ward, 2010), } \\
\text { (Hurt \& Thomas, 2009), } \\
\text { (Duggal, 2009, 2010a), (PMBOK } \\
\text { 5th Edition, 2013) }\end{array}$ \\
\hline
\end{tabular}

Fuente: Elaboración propia.

Nota: Primera parte de la tabla matriz teórica de variables independientes y dependiente 
Tabla 3. Matriz teórica de variables independientes y dependiente

\begin{tabular}{|c|c|c|}
\hline Variables & Definición conceptual & Autores \\
\hline $\begin{array}{l}\text { X7 Aplicar las Lecciones } \\
\text { Aprendidas }\end{array}$ & $\begin{array}{l}\text { Repetir en los proyectos lo que se ha hecho bien } \\
\text { y eliminar lo que se ha hecho mal. }\end{array}$ & $\begin{array}{l}\text { (Duggal, 2009), (Brantley, } \\
\text { 2007), (Hurt \& Thomas, 2009), } \\
\text { (PMI, 2012). }\end{array}$ \\
\hline $\begin{array}{l}\text { X8 Administración de los } \\
\text { Riesgos }\end{array}$ & $\begin{array}{l}\text { Administrar todo evento potencial, que a futuro } \\
\text { puede afectar los resultados del proyecto, que } \\
\text { pueda impactar la factibilidad, recursos y } \\
\text { requerimientos para terminar el proyecto. }\end{array}$ & $\begin{array}{l}\text { (Hurt \& Thomas, 2009), } \\
\text { (Stanleigh, 2009), (Brantley, } \\
\text { 2007), (PMI, 2012). }\end{array}$ \\
\hline $\begin{array}{l}\text { X9 Administración } \\
\text { Control de Cambios }\end{array}$ & $\begin{array}{l}\text { Administrar los requerimientos de cambios en } \\
\text { forma coordinada y metodológica a lo largo de } \\
\text { todo el Proyecto, asegurando que los cambios } \\
\text { estén debidamente analizados y tengan el nivel } \\
\text { de aprobación adecuado. }\end{array}$ & $\begin{array}{l}\text { (Hurt \& Thomas, 2009), } \\
\text { (Stanleigh, 2009), (Brantley, } \\
\text { 2007), (Duggal, 2006), (PMl, } \\
\text { 2012). }\end{array}$ \\
\hline $\begin{array}{l}\text { X10 Posicionamiento } \\
\text { Organizacional }\end{array}$ & $\begin{array}{l}\text { El éxito de la Oficina de Administración de } \\
\text { Proyectos de TI está relacionado con el } \\
\text { aseguramiento de la autoridad necesaria que } \\
\text { debe tener. }\end{array}$ & $\begin{array}{l}\text { (Stanleigh, 2009), (Andersen, } \\
\text { Bjolrn1, Henriksen , Bjolrnar2, } \\
\text { Aarseth y Wenche2m, 2007), } \\
\text { (Ward, 2010), (Hurt \& } \\
\text { Thomas, 2009), (Project } \\
\text { Management Institute, Inc. } \\
\text { PMl.org/Pulse, 2013) }\end{array}$ \\
\hline $\begin{array}{l}\text { Y Generación de Valor } \\
\text { Empresarial (Variable } \\
\text { Dependiente Y) }\end{array}$ & $\begin{array}{l}\text { Definir la generación de valor es difícil; depende } \\
\text { de quién sea el observador, quién la perciba / } \\
\text { reciba. De manera general podría decirse que } \\
\text { significa recibir lo que se espera o que lo recibido } \\
\text { supera las expectativas. El verdadero valor de } \\
\text { las PMO radica en resaltar la relación crítica } \\
\text { entre su trabajo y los resultados del negocio. } \\
\text { Además se puede decir que el Valor de Negocio } \\
\text { de proyectos de TIC se genera cuando se } \\
\text { seleccionan los proyectos adecuados para la } \\
\text { organización, se gestionan de manera } \\
\text { profesional y se ejecutan cumpliendo o } \\
\text { superando las expectativas del cliente. La } \\
\text { selección de estos proyectos implica estimar el } \\
\text { Valor Económico Agregado de cada uno. Este } \\
\text { puede medirse en términos de ahorro en costos, } \\
\text { ingresos adicionales, incremento en la } \\
\text { satisfacción del cliente, cumplir con el ROI } \\
\text { empresarial, etc. }\end{array}$ & $\begin{array}{l}\text { Brantley, W. (2007), Duggal, } \\
\text { S. J. (2009), Hobbs, B. \& } \\
\text { Aubry, M. (2007), Hurt, M. \& } \\
\text { Thomas, J. L. (2009), Kendall, } \\
\text { Gerald I. y Rollins Steven C. } \\
\text { (2003), Kwak, Y. H. \& Xiao Yi } \\
\text { Dai, Ch. (2000), Letavec, } \\
\text { Craig J. (2006), Pinto, A., De } \\
\text { Matheus Cota, M. F. y Levin, } \\
\text { G. (2010), PMI (2014), PMI } \\
\text { (2013), Stanleigh, M. (2009), } \\
\text { Ward, J. L. (2010) }\end{array}$ \\
\hline
\end{tabular}

Fuente: Elaboración propia.

Nota: Segunda parte de la tabla matriz teórica de variables independientes y dependiente 
Figura 1. Modelo gráfico relación de variables

\begin{tabular}{|l|l|l|}
\hline Variables Independientes (Xi) \\
\hline Alineación a los Objetivos Del Negocio \\
\hline Tiempo
\end{tabular}

Fuente: Elaboración propia

\section{Métodos}

Diseño de la investigación

La investigación se realizó bajo un diseño no experimental, del tipo exploratorio, descriptivo, correlacional-causal y explicativo. Es transeccional correlacional-causal, es decir, se describen relaciones entre dos 0 más categorías, conceptos o variables en un momento determinado, permitiendo predecir el comportamiento de una o más variables a partir de otras. (Hernández et al., 2006). La medición de los datos recolectados permite vincular conceptos abstractos con indicadores empíricos. Es no experimental, ya que por la naturaleza del estudio no se manipularon las variables independientes para observar las reacciones en la variable dependiente (Hernández et al., 2006).

Población y muestra

Conforme a las características mencionadas sobre unidad de análisis y delimitaciones, la población de interés para esta tesis son las organizaciones grandes industriales, comerciales y de servicios ubicadas en el estado de 
Nuevo León, lo anterior con base en el criterio del número de recursos humanos empleados, definido por la Secretaría de Economía del país en el Diario Oficial de la Federación del 30 de Junio de 2009. Se contabilizaron 180 organizaciones que son la población de investigación.

Independientemente de la existencia de asociaciones, agrupaciones, cámaras que agrupan a diversas organizaciones y que son una fuente a la cual se puede acudir para seleccionar las organizaciones a encuestar, se decidió utilizar una muestra no probabilística en donde el encuestador selecciona a la muestra representativa, por lo que las organizaciones que se encuestaron fueron en base a la experiencia, contactos y relación profesional de los autores a través de los años. Estas organizaciones pertenecen a diferentes giros y todas cumplen con los requisitos de selección.

El sujeto de estudio son las empresas y se entrevistaron a las personas responsables de ejecutar la gestión de proyectos de TIC.

\section{Datos e instrumentación estadística}

En este estudio, se aplicó: análisis descriptivo y de correlación de las respuestas del cuestionario de las personas encuestadas. La técnica utilizada para realizar esta investigación fue en base a la modelación estadística de pruebas no paramétricas a través de la técnica de modelación mediante ecuaciones estructurales y mínimos cuadrados parciales (SEM-PLS. por sus siglas en inglés), ya que la técnica no asume normalidad en los datos y es conveniente para muestras pequeñas, las recomendaciones mínimas están entre 30 y 100 casos, haciéndose más consistente con muestras grandes (Barclay, Higgins \& Thompson, 1995), además de ser adecuada para predecir y/o generar teorías (Ringle et al., 2009).

La herramienta de software utilizada fue el paquete de cómputo SMART PLS 2.0.M3 (Ringle et al., 2005).

\section{Elaboración de la encuesta}

Se elaboró el cuestionario a utilizar de 35 preguntas y se dividió en tres secciones: 1) Preguntas orientadas a conocer el perfil de la organización que permite la recolección de datos generales, 2) 8 preguntas orientadas a la generación de valor de negocio (preguntas para validar las variables 
independientes con la dependiente) y 3) 27 preguntas para colectar información complementaria. Su diseño ofrece confiabilidad y validez de los datos recolectados. Su diseño ofrece confiabilidad y validez de los datos recolectados.

Los ítems solicitan respuestas en escala Likert de 1 a 5 . De ésta manera se puede medir con mayor objetividad los aspectos relacionados con los constructos y sus variables involucradas.

\section{Validación de la encuesta final}

Como resultado de la validación del primer diseño de encuesta aplicada, se recibió retroalimentación en el sentido de revisar la posibilidad de disminuir aún más el número de preguntas del cuestionario, ya que lo consideraban extenso. Considerando lo anterior y las preguntas clave para el objetivo que se perseguía en la investigación; se re diseñó el cuestionario a utilizar disminuyendo a 35 preguntas, divididas en 2 secciones: 1) Sección preguntas para generar valor de negocio y 2) Sección preguntas información complementaria. Se hizo énfasis en que las preguntas mínimas necesarias a contestar son las de la sección 1 haciendo un total de solo 8 preguntas.

Hipótesis general de investigación

La hipótesis de investigación es la siguiente:

Hi: Validar que la presencia de los factores alineación a objetivos estratégicos, tiempo, presupuesto, alcance, calidad, satisfacción del cliente, lecciones aprendidas, administración del riesgo, control de cambios, posicionamiento organizacional de la $\mathrm{PMO}$, en proyectos de tecnología de información y comunicaciones, son los que generan valor de negocio por medio de la gestión realizada por la oficina de administración de proyectos de TIC (PMO, por sus siglas en Inglés) o de la administración de proyectos de TIC (cuando la organización no tiene una PMO formal). 


\section{Resultados}

Representación gráfica del modelo de estudio

El modelo SEM utilizado en el estudio con sus respectivos valores es el siguiente:

Figura 2. Resumen de resultados mostrando los modelos interno y externo, con la $R^{2}$, Betas y cargas estandarizadas. Algoritmo PLS

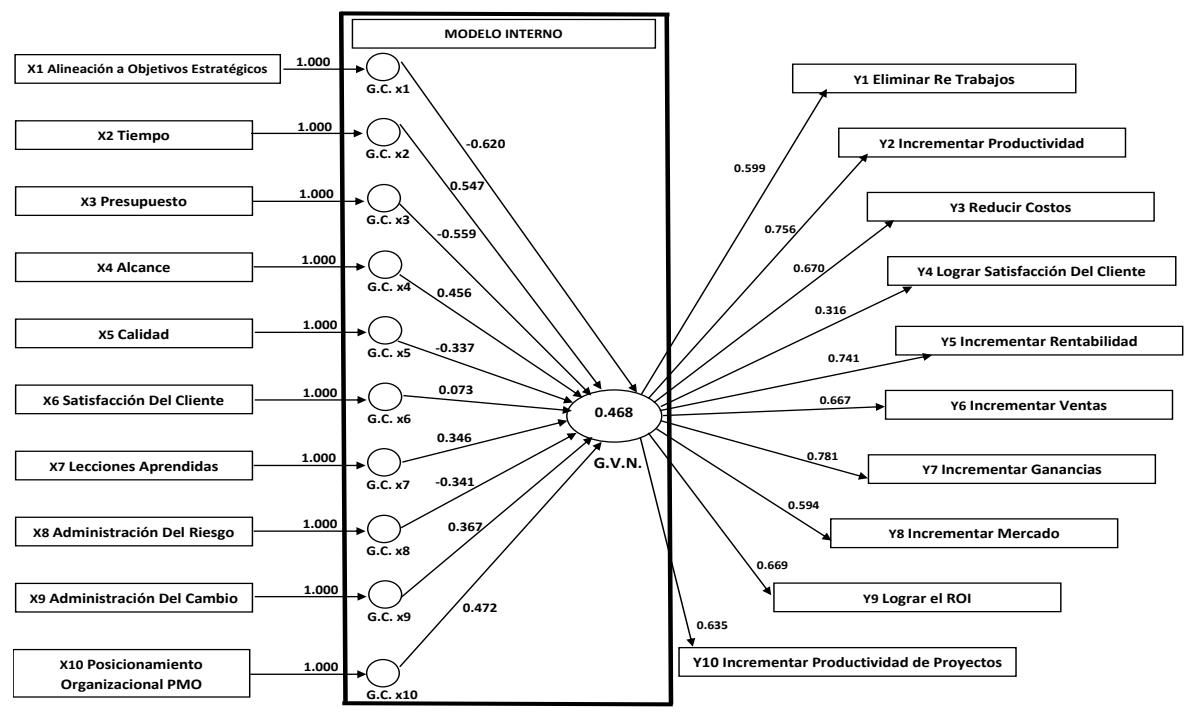

Fuente: Elaboración propia. Corrida del SmartPLS 2.0.M3 (Ringle et al., 2005)

La representación gráfica de la evaluación del modelo interno (Inner Model) - estructural y externo (Outer Moder) - Medición. Algoritmo Bootstrapping 5000 muestras, es la siguiente: 
Figura 3. Resultados del modelo mostrando los valores $t$ de las variables e indicadores.

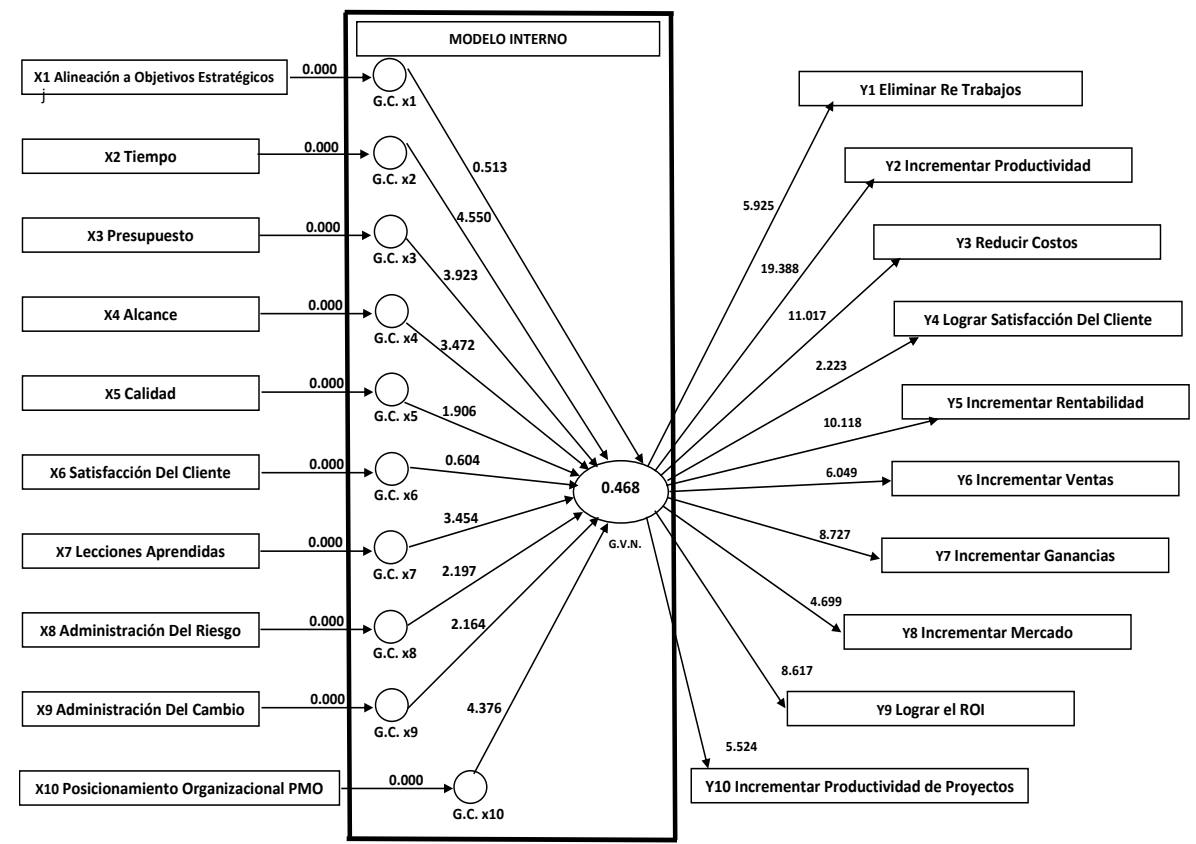

Fuente: Elaboración propia. Corrida del SmartPLS 2.0.M3 (Ringle et al., 2005)

\section{Tabla 4. Criterios de Calidad del Modelo PLS-SEM .Resumen resultados ejecución en SmartPLS (35 casos).}

\begin{tabular}{lcccc}
\hline \multicolumn{1}{c}{ Constructo } & AVE & Composite reliability & R Square & Cronbachs alpha \\
\hline X1 Alineación a Objetivos Estratégicos & 1.000000 & 1.000000 & & 1.000000 \\
X2 Tiempo & 1.000000 & 1.000000 & & 1.000000 \\
X3 Presupuesto & 1.000000 & 1.000000 & & 1.000000 \\
X4 Alcance & 1.000000 & 1.000000 & & 1.000000 \\
X5 Calidad & 1.000000 & 1.000000 & & 1.000000 \\
X6 Satisfacción del Cliente & 1.000000 & 1.000000 & & 1.000000 \\
X7 Lecciones Aprendidas & 1.000000 & 1.000000 & & 1.000000 \\
X8 Administración del Riesgo & 1.000000 & 1.000000 & & 1.000000 \\
X9 Administración del Cambio & 1.000000 & 1.000000 & & 1.000000 \\
X10 Posicionamiento Organizacional & 1.000000 & 1.000000 & & 1.000000 \\
Y Generar Valor de Negocio & 0.428732 & 0.878543 & 0.467812 & 0.843022 \\
\hline
\end{tabular}

Fuente: Elaboración propia. Corrida del SmartPLS 2.0.M3 (Ringle et al., 2005) 
Tabla 5. Resumen resultados del modelo seleccionado mostrando la $R^{2}$, betas y cargas estandarizadas. Algoritmo PLS. $\mathrm{R}^{2}=0.468$

\begin{tabular}{lcclc}
\hline \multicolumn{1}{c}{ Variable observada } & $\begin{array}{c}\text { Modelo } \\
\text { interno }\end{array}$ & Impacto & \multicolumn{1}{c}{ Variable medida } & Modelo externo \\
\hline X1 Al_Obj_Est & -0.620 & & Y1 Eliminar. Re trabajo & 0.599 \\
X2 Tiempo & 0.547 & Muy fuerte & Y2 Incrementar Productividad & 0.756 \\
X3 Presupuesto & -0.559 & & Y3 Reducción Costos & 0.670 \\
X4 Alcance & 0.456 & Fuerte & Y4 Satisfacción. Del Cliente & 0.316 \\
X5 Calidad & -0.337 & & Y5 Incrementar Rentabilidad & 0.741 \\
X6 Satisfacción del Cliente & 0.073 & Imperceptible & Y6 Incrementar Ventas & 0.667 \\
X7 Lecciones Aprendidas & 0.346 & Fuerte & Y7 Incrementar Ganancias & 0.781 \\
X8 Administración del Riesgo & -0.341 & & Y8 Incrementar Mercado & 0.594 \\
X9 Administración del Cambio & 0.367 & Fuerte & Y9 Lograr ROI & 0.669 \\
X10 Posicionamiento Org. PMO & 0.472 & Fuerte & Y10 Incrementar Prod. Proy. & 0.635 \\
\hline
\end{tabular}

Fuente: Elaboración propia. Corrida del SmartPLS 2.0.M3 (Ringle et al., 2005)

Tabla 6. Modelo interno y externo. Resumen de valores de variables observadas. Algoritmo Bootstrapping. Valores $T$.

\begin{tabular}{lcc}
\hline \multicolumn{1}{c}{ Variable observada } & $\begin{array}{c}\text { Valor del coeficiente con 5000 } \\
\text { muestras. Modelo interno. } \\
\text { Valores T }\end{array}$ & $\begin{array}{c}\text { Valor del coeficiente con 5000 } \\
\text { muestras. Modelo externo. } \\
\text { Valores T }\end{array}$ \\
\hline X1 Alineación a Objetivos Estratégicos & 0.513 & 5.925 \\
X2 Tiempo & 4.550 & 19.388 \\
X3 Presupuesto & 3.923 & 11.017 \\
X4 Alcance & 3.472 & 2.223 \\
X5 Calidad & 1.906 & 10.118 \\
X6 Satisfacción Del Cliente & 0.604 & 6.049 \\
X7 Lecciones Aprendidas & 3.454 & 8.727 \\
X8 Administración Del Riesgo & 2.197 & 4.699 \\
X9 Administración Del Cambio & 2.164 & 8.617 \\
X10 Posicionamiento Organizacional PMO & 4.376 & 5.524 \\
\hline
\end{tabular}

Fuente: Elaboración propia. Corrida del SmartPLS 2.0.M3 (Ringle et al., 2005)

\section{Discusión de resultados}

\section{Alpha de Cronbach}

A continuación se presenta una discusión sobre la fiabilidad del modelo, así como la interpretación de los mismos.

Fiabilidad. Como es sabido, el alpha de cronbach se incrementa en la medida en la que la correlación entre los ítems se incremente. Es por ésta razón que se utiliza este método para medir la consistencia interna del 
cuestionario, de la prueba. Su máximo valor es 1 y su mínimo es 0 aunque puede ser negativo. Una regla comúnmente aceptada es que un alpha de 0.7 (incluso 0.6) indica una confiabilidad aceptable y un 0.8 o mayor indica una buena confiabilidad. Fornell \& Larcker (1981). Por convención, se aplican los mismos puntos de corte, o criterios de calidad, establecidos para la medición de la Confiabilidad Compuesta. (Garson, 2016, p. 63, p. 64).

- Mayor o Igual a .80 para una buena escala (Daskalakis \& Mantas, 2008, p. 288).

- .70 para una escala aceptable (Henseler, Ringle, \& Sarstedt, 2012, p. 269).

- 60 es aceptable para una escala para propósitos exploratorios (Chin, 1998; Höck \& Ringle, 2006, p. 15).

Conforme se muestra en la Tabla 4 Criterios de calidad del modelo PLS-SEM. Resumen resultados ejecución en SmartPLS, después de realizada la evaluación se pudo determinar que el instrumento de medición utilizado para esta investigación es confiable ya que muestra en su mayoría niveles aceptables de Alfa Cronbach mostrando un valor de 1 en todos los indicadores, es decir, son medidos en un nivel de buena escala para la investigación exploratoria. Esto indica una alta correlación de los ítems y no necesariamente que pudiera existir redundancia.

Se utilizó el paquete de cómputo SMART PLS 2.0.M3 (Ringle et al., 2005) para la obtención de las cargas estandarizadas de los indicadores, ya que por la naturaleza de las interrelaciones entre variables independientes y dependientes, el empleo de Alfa de Cronbach no es definitorio, debido a que analiza la relación de los ítems con los constructos de manera aislada, en tanto que SMARTPLS (Ringle et al., 2005) los analiza considerando el efecto combinado de los ítems en todos los constructos, (Monge, 2014).

\section{Confiabilidad compuesta}

Los modelos reflexivos deben ser evaluados en base a la confiabilidad y validez de los constructos, para estos efectos se usa la medición confiabilidad compuesta (composite reliability, en inglés) como un estimado de la consistencia interna del constructo. El criterio de calidad de Hair, Ringle \& Sarstedt, (2011) dice que los valores de la medición deben ser mayores a 0.70 . 
Otros criterios de calidad se mencionan a continuación: Para modelos con fines exploratorios, la confiabilidad compuesta debe ser igual o mayor de 6 (Chin, 1998; Höck \& Ringle, 2006, p. 15). Para modelos con fines confirmatorios, igual o mayor que .70 (Henseler, Ringle, \& Sarstedt, 2012, p. 269). Para investigaciones confirmatorias, igual o mayor que .80 (Daskalakis \& Mantas, 2008, p. 288).

La validez en los modelos de medición reflexivos se enfoca en la validez convergente a través de la varianza extraída media (AVE, por sus siglas en inglés) se mide la validez convergente, La confiabilidad compuesta es una alternativa preferida al alfa de Cronbach como una prueba de la validez convergente en un modelo reflexivo. Se puede preferir como medida de fiabilidad porque el alfa de Cronbach puede sobrevalorar o subestimar la fiabilidad de la escala, usualmente la última. Comparado con el alfa de Cronbach, la confiabilidad compuesta puede conducir a mayores estimaciones de confiabilidad verdadera. El punto de corte aceptable para la confiabilidad compuesta es el mismo que para cualquier medida de confiabilidad, incluyendo alfa de Cronbach. (Garson, 2016, p.63). La confiabilidad compuesta varía de 0 a 1 , siendo 1 la fiabilidad estimada perfecta, la cual debe mostrar valores mayores a 0.50 (Fornell \& Larcker, 1981).

Como se puede ver en la Tabla 4 Criterios de calidad del modelo PLSSEM. Resumen resultados ejecución en SmartPLS, que en el caso del presente estudio la fiabilidad compuesta (Fornell \& Larcker, 1981) es superior a 0.8 en todos los constructos, superando el umbral más exigente propuesto por Nunnally (1978) e indicando consistencia interna en los constructos del modelo, es decir un buen grado de validez convergente, lo que significa que una determinada variable latente explica más de la mitad de la varianza que sus indicadores (Hair et al., 2011). Ante la posible interrogante de que los valores altos obtenidos (>.90) pudieran indicar que los indicadores múltiples varían solo de redacción en lugar de ser medidas verdaderamente representativas del constructo que representa el factor, se considera que los indicadores si son representativos de la dimensión deseada y simplemente se correlacionan altamente como lo mencionan Hair, Hult, Tomas, Ringle, Christian \& Sarstedt, (2014). 


\section{Modelo externo - medición}

Respecto de la validación de la fiabilidad de los ítems, la importancia y significancia relativa del factor de carga resultó ser importante y que puedan ser considerados en la composición de las variables, considerando que los valores obtenidos en 9 de las 10 variables $(0.599,0.756,0.670,0.741,0.667$, $0.781,0.594 .0 .669,0.635)$ reflejan una carga en el factor mayor al 0.5 , Hair, Anderson, Tatham \& Black (1999), como se puede observar en los resultados del modelo externo en la Tabla 5 Resumen resultados del modelo seleccionado mostrando la $\mathrm{R}^{2}$, Betas y cargas estandarizadas, así como en la Figura 2. Resumen Resultados del Modelo mostrando Modelo Interno y Modelo Externo, con la $\mathrm{R}^{2}$, Betas y Cargas Estandarizadas, y por lo tanto se consideran aceptados.

No se eliminó ningún ítem ya que nueve de diez indicadores no son inferiores a 0.50 (Hair et al., 2011), todos son significativos a un nivel de confianza del $95.0 \%$, como se puede observar en los resultados que aparecen en la Tabla 6 Modelo Interno y Externo Resumen valores de variables observadas. Algoritmo Bootstrapping, y muestran una validez convergente superior a 0.5 (Fornell \& Larcker, 1981).

La fiabilidad compuesta (Fornell \& Larcker, 1981) es superior a 0.8 en todos los constructos, superando el umbral más exigente propuesto por Nunnally (1978) e indicando consistencia interna en los constructos del modelo, es decir un buen grado de validez convergente, como se puede observar en los resultados que aparecen en la Tabla 4 Criterios de Calidad del Modelo PLS-SEM .Resumen resultados ejecución en SmartPLS.

\section{Modelo interno - estructural}

En la evaluación del modelo interno se realizaron varias ejecuciones para encontrar la que generara la mayor $\mathrm{R}^{2}$. Se decidió no eliminar ninguna variable causal así como ninguna variable medida, ya que su combinación conjunta generó la $R^{2}$ más alta siendo ésta 0.468 clasificándose de un efecto o fuerza moderada de acuerdo a los criterios establecidos por Chin (1998, p. 323; Höck \& Ringle, 2006, p. 15). Ver Figura 2. Resumen Resultados del Modelo mostrando Modelo Interno y Modelo Externo, con la $R^{2}$, Betas y Cargas Estandarizadas. Algoritmo PLS 
El diagnóstico del nivel de significancia de los path coefficients fue utilizando el algoritmo PLS Bootstrapping con 5000 muestras obteniendo resultados significativos de los valores T del modelo estructural, en 8 de 10 variables, con valores igual o superior a 1.96 (nivel de significancia $=0.050$ $5 \%$ ), como se puede ver en la Tabla 6 Modelo Interno y Externo. Resumen valores de variables observadas. Algoritmo Bootstrapping.

Se utilizó el Algoritmo PLS Bootstrapping con 5,000 muestras para la medición del nivel de significancia de las variables del modelo estructural y el de medición. Los resultados obtenidos muestran valores significativos de $t$ por arriba de 1.96. En el modelo estructural aparecen 2 variables con valor por debajo de 1.96. Alineación a objetivos estratégicos con 0.513 y Satisfacción del cliente con 0.604 , no obstante lo anterior se decide conservar en el modelo esas dos rutas por razones de su importancia teórica.

Para la relevancia predictiva $Q^{2}$ se utilizó PLS Blindfolding para la medición de la capacidad predictiva del modelo estructural, Stone-Geisser $Q^{2}$ (Geisser, 1974; Stone, 1974), tanto para Construct Cross Validated Redundancy como para Construct Cross Validated Communality. El resultado obtenido muestra una relevancia predictiva del factor endógeno clasificada como moderada por el método de redundancia, y alto por el de comunalidad. Lo anterior de acuerdo a los resultados obtenidos comparados con los que se muestran en las tablas 7 y 8 ; y que conforme al criterio de calidad de Cohen (1988), el valor .02 representa un tamaño de efecto "pequeño o débil", .15 representa un tamaño de efecto "medio o moderado", y .35 representa un tamaño de efecto "alto o substancial".

\section{Porqué no se eliminan indicadores}

En la definición de un modelo no deben excluirse variables importantes desde el punto de vista teórico. En primer lugar, debe hacerse un esfuerzo por medir todas las variables pertinentes. Basado en la experiencia profesional, en el tema de la presente investigación, todas las variables se consideran importantes y altamente significativas, hablando de su importancia teórica.

Para los modelos formativos (variables $\mathrm{x}$ ), sin embargo, cada indicador mide uno de los conjuntos de dimensiones de las que se compone el factor, dicho de otra manera, cada indicador representa una dimensión del 
significado de la variable latente. Eliminar tal indicador equivale a eliminar una dimensión del significado, ocasionando que el significado de la variable latente cambie porque se omite esa dimensión del significado. Por lo tanto, a menos que haya elementos indicadores redundantes para la dimensión en cuestión, situación que no existe en el modelo, los indicadores generalmente no se eliminan de los modelos formativos aunque no sean significativos (Garson, 2016, p. 103).

Como se pudo observar en los resultados obtenidos de las diferentes corridas, eliminar los coeficientes con valor negativo no impacta favorablemente al valor obtenido de la $\mathrm{R}^{2}$, si recordamos la importancia de tener una $\mathrm{R}^{2}$ con el valor más alto posible con objeto de que el modelo tenga un poder más alto de explicación, en otras palabras, que la varianza en la variable endógena generar valor de negocio sea explicada lo más alto posible por el modelo. El valor más alto de la $\mathrm{R}^{2}$ se obtuvo en el modelo que conjunta las 10 predictoras, aun considerando el efecto del valor negativo que muestran algunas de las variables exógenas con el efecto del valor positivo que muestran otras.

\section{Determinación de la $R^{2}$}

El valor de la $\mathrm{R}^{2}$ refleja la magnitud de las relaciones estructurales entre los constructos exógenos y los endógenos. Representa el poder explicativo del modelo sobre los constructos endógenos. Otra forma de expresarlo es que $\mathrm{R}^{2}$, también llamado coeficiente de determinación, es la medida del tamaño del efecto global para el modelo estructural / interno, como en la regresión, indicando a continuación que el $46.8 \%$ de la varianza en la variable generar valor de negocio se explica por el modelo. Es importante buscar la $\mathrm{R}^{2}$ con el valor más alto con objeto de lograr la mayor explicación del modelo. Para ésta investigación se seleccionó la $\mathrm{R}^{2}$ obtenida de $46.8 \%$ por ser el valor más alto obtenido de las corridas ejecutadas, siendo de una fuerza o efecto moderado de acuerdo a las clasificaciones siguientes: 
Tabla 7. Criterio de calidad fuerza del coeficiente de determinación o $R^{2}$

\begin{tabular}{ccc}
\hline Criterio de calidad & Clasificación & Referencia \\
\hline$>0.19$ & Débil & Chin (1998, p. 323); Höck \& Ringle (2006, p. 15) \\
$>0.33$ & Moderada & Chin (1998, p. 323); Höck \& Ringle (2006, p. 15) \\
$>0.67$ & Substancial & Chin (1998, p. 323); Höck \& Ringle (2006, p. 15) \\
\hline
\end{tabular}

Fuente: Elaboración propia

Tabla 8. Criterio de calidad fuerza del coeficiente de determinación o $R^{2}$

\begin{tabular}{ccc}
\hline Criterio de calidad & Clasificación & Referencia \\
\hline$>0.25$ & Débil & (Hair et al., 2011) \\
$>0.50$ & Moderada & (Hair et al., 2011) \\
$>0.75$ & Substancial & (Hair et al., 2011) \\
\hline
\end{tabular}

Fuente: Elaboración propia

\section{Conclusiones, implicaciones y recomendaciones}

\section{Conclusiones principales}

Se explican detalladamente las diferentes aportaciones, logradas con esta investigación, hechas al conocimiento, al identificar las diferentes formas de generar valor de negocio como son: eliminar re trabajos, reducir costos, lograr satisfacción del cliente, lograr el ROI, incrementar productividad, rentabilidad, ventas, ganancias, mercado, productividad de proyectos, y por otro lado aquellas variables que requieren una especial atención en la gestión de los proyectos de tecnología de información y comunicaciones: alineación a objetivos estratégicos, tiempo, presupuesto, alcance, calidad, satisfacción del cliente, lecciones aprendidas, administración del riesgo, administración control de cambios, posicionamiento organizacional PMO TIC .

El modelo utilizado en la investigación puede aplicarse para una PMO TIC de cualquier organización y de cualquier giro, que tenga el interés de evolucionar a ser una unidad que genere valor de negocio.

Un hallazgo importante encontrado fue la alta apatía de los encuestados para participar en un estudio de investigación.

Por otro lado es interesante encontrar una diferencia entre el nivel de importancia que los administradores de proyecto otorgan a la gestión de las variables causales, lograr la satisfacción del cliente, alineación de los 
proyectos a los objetivos estratégicos de la organización, calidad, Presupuesto y lo que la literatura consultada y juicios de expertos declaran y recomiendan. Esto representa un área de oportunidad altamente importante para culturizar a dichos actores.

La gestión de las variables tiempo, alcance se reflejan de alta importancia para los administradores de proyectos, lo cual es un punto de coincidencia con lo que aparece en la literatura consultada.

Contrario a la expectativa sobre los resultados, la gestión de las variables posicionamiento organizacional de la PMO, lecciones aprendidas, administración del control de cambios, muestran una valoración de impacto de nivel fuerte. Sin embargo, después de analizar detenidamente lo que significan dichas variables, se encontró que existen razones de peso para que hayan sido consideradas importantes por los administradores de proyectos de TIC. Más explicación al respecto en el desarrollo detallado del punto Implicaciones Prácticas.

Un resultado más de este estudio lo constituyen las diferentes líneas de investigación que se identificaron y se mencionan a detalle.

Se propone la sensibilización / culturización a los administradores de proyectos de tecnología de información y comunicaciones, para que en el desempeño de su gestión visualicen y traduzcan los resultados de los proyectos en resultados del negocio.

\section{Principales aportaciones al conocimiento}

Originalidad y valor. Para esta investigación se construyó y realizaron pruebas de un modelo utilizando la técnica de análisis basada en la modelación estadística de pruebas no paramétricas, mediante ecuaciones estructurales basada en varianza y mínimos cuadrados parciales (PLS-SEM, por sus siglas en inglés), ya que la técnica no asume normalidad en los datos y es conveniente para muestras pequeñas (20, 30 observaciones), además de ser adecuada para predecir y/o generar teorías (Ringle et al., 2009), que integra y mide en una sola variable latente (Generar valor de negocio) el impacto de las variables observadas: alineación a objetivos estratégicos, tiempo, presupuesto, alcance, calidad, satisfacción del cliente, lecciones aprendidas, administración del riesgo, administración del cambio, posicionamiento organizacional del área de gestión de proyectos de TIC, el 
modelo está basado en un análisis realizado a la literatura de las teorías relacionadas con la gestión de las variables mencionadas, lo cual constituye una aportación y avance en el conocimiento y práctica profesional de la gestión de proyectos de tecnología de información y comunicaciones, al considerar los efectos conjuntos de las variables mencionadas, esto es importante en el contexto de nuestro país México y específicamente del estado de Nuevo León.

Lo anterior representa una contribución importante al conocimiento en el tema de la gestión de proyectos de tecnología de información y comunicaciones, al detallar y describir las diferentes variables, que por su comportamiento encontrado en el modelo, deben ser gestionadas con especial y mayor atención, también resulta altamente importante la aportación de las diferentes formas en las que se puede medir la generación de valor de negocio. Por lo tanto, este modelo puede utilizarse como herramienta para la mejora continua en el desempeño de las áreas responsables de la gestión de proyectos de tecnología de información y comunicaciones en las organizaciones.

El modelo de la investigación puede ser empleado por cualquier organización pública o privada, sea cual sea el giro al que se dedique, como un instrumento que pudiera servir de base para evaluar el desempeño de las áreas responsables de la gestión de proyectos de tecnología de información y comunicaciones y derivar acciones en consecuencia. Comentando sobre la evolución de los modelos de ecuaciones estructurales; PLS se ha extendido a la investigación en educación, mercadotecnia, psicología, y las ciencias sociales (Jacobs et al., 2011). En este sentido, el presente trabajo se incorpora al número de estudios que se han realizado y que consta en la literatura moderna en journals internacionales, en materia de aplicación de ecuaciones estructurales PLS-SEM, en el tema de la gestión de proyectos de tecnología de información y comunicaciones.

Hallazgos importantes. El primer hallazgo importante que se quiere resaltar es el haber encontrado una apatía muy alta en los encuestados para participar, misma que se vio reflejada en: 1) Simplemente no participar, 2) Los que si participaron, presentaron un alto tiempo de respuesta para enviar sus cuestionarios ya contestados, esto último aun y dando un estrecho seguimiento con todos, la anterior fue una situación que nunca se pensó que sucedería, sobre todo, considerando el nivel profesional de los involucrados y 
el supuesto de que trabajan en un tema que los apasiona, así como el tema de investigación. En relación al segundo hallazgo, y analizando los resultados del estudio en lo referente al modelo interno, muestran los siguientes aspectos muy importantes, basados en las respuestas generadas por los encuestados y clasificando la valoración del impacto en base a la guía integrada por el Dr. Juan Rositas (2015), ver Tabla 9 Coeficientes y su impacto en el modelo: Una variable predictora con un valor muy fuerte: Tiempo 0.547, cuatro variables predictoras aparecen con un valor fuerte: Posicionamiento organizacional del área de gestión de proyectos de TIC 0.472, Alcance 0.456, Administración del cambio 0.367, Lecciones aprendidas 0.346. Estos valores pueden observarse en la tabla 17 Modelo Interno. Resumen Valoración del Impacto de los Coeficientes. Algoritmo PLS.

Tabla 9. Coeficientes y su impacto en el modelo

\begin{tabular}{cccc}
\hline \multicolumn{2}{c}{ Rango para coeficientes } & Valoración de impacto \\
\hline 0.00 & a & 0.09 & Imperceptible \\
0.10 & a & 0.15 & Perceptible (apenas) \\
0.16 & a & 0.19 & Considerable \\
0.20 & a & 0.29 & Importante \\
0.30 & a & 0.50 & Fuerte \\
Mayores a & 0.50 & Muy Fuerte \\
\hline
\end{tabular}

Fuente: Guía integrada por Rositas (2015).

Significando lo anterior que tienen un impacto importante y estadísticamente significativo en la generación de valor de negocio (G.V.N.), impactando este último constructo latente, a significativos indicadores claves para el negocio como lo son: eliminar re trabajos, reducir costos, lograr satisfacción del cliente, lograr el ROI, incrementar productividad, rentabilidad, ventas, ganancias, mercado, productividad de proyectos

Una variable predictora, Lograr la satisfacción del cliente, con un valor imperceptible 0.073 . Cuatro variables predictoras con valores negativos: Alineación a objetivos estratégicos -0.620 , presupuesto -0.559 , Administración de riesgos -0.341 , Calidad -0.337 . Aun siendo negativos se decidió no eliminarlos debido a las siguientes razones: Importancia teórica que representan, cada indicador representa una dimensión que define el significado de la variable latente; al eliminarlo equivaldría a cambiar el significado de dicha variable, el valor más alto de la $R^{2}$ se genera 
conjuntamente por el valor de todas las variables exógenas; considerando el efecto de los valores positivos y los negativos, el eliminar cualquiera de las variables con valor negativo impacta desfavorablemente, disminuyendo el valor generado de $\mathrm{R}^{2}$. Por lo tanto, a menos que haya elementos indicadores redundantes para las dimensiones en cuestión, situación que no existe en el modelo, los indicadores generalmente no se eliminan de los modelos formativos aunque no sean significativos (Garson, 2016, p. 103).

Los valores de la t fueron significantes en al menos 95\%. Los análisis detallados de lo descrito en las anteriores líneas fue previamente presentado en la secciones de resultados y discusión.

En este análisis, después de haber comparado la realidad de lo que piensan y ejecutan las personas responsables de gestionar proyectos de tecnología de información y comunicación, con las teorías más actuales recomendadas por organismos y profesionales expertos en el tema que se consigna en la diversa literatura consultada, se encontraron diferencias en relación a lo que la teoría recomienda; lo anterior significa que, dichos profesionales deben trabajar con mayor dedicación / esfuerzo para adoptar y alinearse continúa y decididamente a dichas recomendaciones, ampliar su visión de que existen otros factores que contribuyen fuertemente al éxito de los proyectos de TIC.

Otras investigaciones. Las conclusiones de este estudio pueden generalizarse a todo tipo de organización pública o privada, en todo el país, sea cual sea el giro al que se dedique, buscando con ello enriquecer esta investigación aplicando el modelo a otras organizaciones.

\section{Aportación práctica}

Recientemente propusimos implementar el modelo para generar valor de negocio por una PMO, estudiado y presentado en esta investigación, en un Grupo Financiero de los más grandes en México, como faro que guíe la actuación de la Oficina corporativa de administración de programas y proyectos de TIC, y también de proyectos que no son tecnológicos. El propósito es proveerle de un sello distintivo, que la diferencie de otras similares en la ejecución de su desempeño, en donde su objetivo principal sea generar valor de negocio utilizando el modelo presentado. Como se ha comentado con anterioridad, será importante identificar y definir las formas de 
vincular la actuación de ésta unidad organizacional, con el logro del objetivo mencionado a través de una gestión profesional y más estricta de los proyectos. Se ha avanzado en sensibilizar a personal clave sobre la adopción de la propuesta, en definir su planeación estratégica, la definición y declaración de su Misión y Visión, los objetivos estratégicos y operativos por alcanzar, así como el camino a seguir.

A la fecha de la presentación de este documento, el modelo propuesto fue aceptado y ya se está trabajando en normar los procesos de esta oficina, dentro de los cuáles se incorpora el modelo de investigación para que esta unidad organizacional genere valor de negocio.

\section{Líneas de investigación futuras}

Por otra parte resultan interesantes otras líneas de investigación derivadas de la presente; como pueden ser:

1) Definir las principales causas por las que los proyectos se retrasan.

2) Determinar los tipos de PMO existentes en las organizaciones Mexicanas de acuerdo a su enfoque (Táctico, Operacional, Estratégico) y a su alcance (Empresarial, Departamental).

3) Definir las principales actividades / Funciones que realizan las PMO en las organizaciones Mexicanas.

4) Identificar formas de traducir los resultados del proyecto en resultados de negocio.

5) Definir factores críticos para medir el desempeño de los project managers.

6) Determinar las razones por las que se generan proyectos fallidos.

7) Describir estructura, características, valorización de proyectos excedidos en tiempo y/o costo.

8) Identificar a profundidad las causas de una débil gestión de las variables que resultaron con valor imperceptible y negativo en la presente investigación.

9) Identificar las causas por las que los administradores de proyectos de TIC asignan mínima importancia a la gestión de variables tan importantes como: alineación a objetivos estratégicos del negocio, lograr la satisfacción del cliente, calidad, presupuesto, administrar los riesgos. 


\section{Implicaciones prácticas}

En la Tabla 10 se presenta el resumen de valoración de impacto de los coeficientes de las variables. En relación a las variables predictoras tiempo y alcance, cuya valoración de impacto fue Muy Fuerte y Fuerte respectivamente de acuerdo con la guía integrada por Rositas (2015), (ver Tabla 9), existe coincidencia con lo que consta en la literatura consultada la cual encuentra que dentro de los primeros lugares catalogados por las organizaciones investigadas, estas dos variables son consideradas como factores que están presentes en proyectos exitosos (Stanleigh, 2008, Duggal, 2010a, Ward, 2010).

Tabla 10. Modelo interno. Resumen de valoración del impacto de los Coeficientes. Algoritmo PLS. Ordenadas de mayor a menor

\begin{tabular}{lcc}
\hline \multicolumn{1}{c}{ Variable Observada } & Valor del Coeficiente & $\begin{array}{c}\text { Valoración del } \\
\text { impacto }\end{array}$ \\
\hline X2 Tiempo & 0.547 & Muy Fuerte \\
X10 Posicionamiento Organizacional PMO & 0.472 & Fuerte \\
X4 Alcance & 0.456 & Fuerte \\
X9 Administración Del Cambio & 0.367 & Fuerte \\
X7 Lecciones Aprendidas & 0.346 & Fuerte \\
X6 Satisfacción Del Cliente & 0.073 & Imperceptible \\
X5 Calidad & -0.337 & \\
X8 Administración Del Riesgo & -0.341 & \\
X3 Presupuesto & -0.559 & \\
X1 Alineación a Objetivos Estratégicos & -0.620 & \\
\hline
\end{tabular}

Fuente: Elaboración propia. Corrida del SmartPLS 2.0.M3 (Ringle et al., 2005)

Con respecto a la variable predictora Posicionamiento organizacional de la PMO TIC, lo que los encuestados manifiestan en esta investigación, es que al otorgarle a esta oficina una alta autoridad organizacional y funcional, le permitiría una mayor efectividad en la culturización a todo el personal involucrado para realizar una gestión profesional de proyectos de tecnología de información y comunicaciones orientada a la generación de valor de negocio.

Lo anterior toma relevancia al recordar que uno de los principales propósitos de una oficina de administración de proyectos de TIC es contribuir a cambiar las prácticas del tema en la organización. Para lograrlo, la Oficina y 
sus recursos dependen de tener una real autoridad organizacional para así tener un impacto o influencia en la gestión de los proyectos de TIC.

Hablando de la variable predictora lecciones aprendidas, se puede considerar de alto impacto ya que al implicar una documentación formal y, sobre todo, capitalización para futuros proyectos, de todo lo realizado en anteriores, de lo que se hizo bien, funcionó bien y generó buenos resultados; además de lo que no se hizo bien, no funcionó bien y generó malos resultados, o de lo que pudo haberse hecho mejor, el llevar una adecuada gestión de esta variable permite focalizarse en mejorar la ejecución de los proyectos derivando en beneficios como un alto grado de proyectos exitosos y así poder avanzar en la consecución de generar valor de negocio.

Por otro lado, es preocupante que la variable lograr la satisfacción del cliente haya obtenido un impacto de imperceptible, reflejando esto una absoluta falta de conocimiento o una muy preocupante ausencia dentro de sus prioridades, por parte de los responsables y ejecutores de administrar los proyectos, de la gran importancia que tiene no solo para el éxito del proyecto sino para el negocio mismo.

De las diferencias mencionadas en hallazgos importantes y específicamente hablando de 3 variables predictoras que obtuvieron resultados negativos: alineación a objetivos estratégicos, presupuesto y calidad y una que obtuvo un valor imperceptible: lograr la satisfacción del cliente, los cuatro son identificadores que en la literatura consultada se menciona de alta importancia para el éxito de los proyectos de tecnología de información y comunicaciones y en consecuencia la generación de valor para las organizaciones, y sin embargo los encuestados no las consideran importantes. La interpretación de lo que están diciendo es que el efecto negativo que tienen sobre la variable latente indica que un menor nivel de alineación a objetivos estratégicos, presupuesto, calidad y administrar los riesgos permite predecir un mayor nivel para generar valor de negocio, lo cual es contradictorio a lo que manifiesta la literatura consultada. A continuación comentarios importantes al respecto:

Alineación a objetivos estratégicos: El Project Management Institute menciona que en base a sus investigaciones anuales que realiza entre las organizaciones; ésta es la principal función de una PMO que con mayor claridad puede aportar / generar valor a la organización. 
Lograr la satisfacción de los clientes: Como lo comenta Ward (2010) al asegurar el logro de los requerimientos del proyecto e incrementar la satisfacción de los clientes es lógico que la organización vea mejoras en áreas como incremento en la participación de mercado, las utilidades, el ingreso, decremento en los costos, siendo éstos temas indicadores de generación de valor para el negocio.

Presupuesto: El Project Management Institute y diferentes investigadores mencionados con anterioridad; declaran que en sus investigaciones las organizaciones encuestadas han manifestado que ésta variable está presente en proyectos que han sido exitosos. Por otra parte dentro de las principales funciones de la PMO se encuentra importantemente relacionada con el aseguramiento en el cumplimiento del presupuesto del proyecto.

Calidad: El Project Management Institute y diferentes investigadores mencionados con anterioridad; también declaran que en sus investigaciones las organizaciones encuestadas han manifestado que ésta variable está presente en proyectos que han sido exitosos.

Algo que debe preocupar a las organizaciones es lo que está sucediendo con el personal responsable de dirigir, administrar y ejecutar la gestión de proyectos de tecnología de información y comunicaciones, que en la gestión de estas variables no las perciben como causales importantes para generar valor de negocio, no las consideran en su dimensión correcta o les asignan mínima importancia dentro de los objetivos del ejercicio de su profesión. Tal vez podría deberse a sobrecarga de trabajos / proyectos, imposición de fechas de terminación, facilidad para conseguir los recursos financieros para los proyectos, una débil cultura de traducir los resultados de los proyectos en resultados para el negocio, ésta resulta ser una interesante línea adicional de investigación.

Con base en la experiencia profesional de los autores; la situación anterior se ha constatado en diferentes organizaciones de la localidad en donde se ha laborado, adicionalmente se considera que para las organizaciones mexicanas no está claramente definida la forma de cómo medir la actuación de las PMO TIC, o de las áreas responsables de gestionar los proyectos de tecnología de información y comunicaciones, y en consecuencia tampoco la medición del desempeño de los administradores de 
proyectos, desde la óptica de generación de valor para la organización; y tampoco está implementada la práctica de la misma.

Con respecto a las diferentes formas de medir la generación de valor de negocio, se muestra a continuación la Tabla 11 con el resumen con los valores generados clasificando su valoración conforme al criterio de calidad de Chin (1998, p. 323); Höck \& Ringle (2006, p. 15) para medir la valoración de la $R^{2}$, en base a los resultados obtenidos de la investigación. Lo anterior considerando que estas variables constituyen diferentes formas en que la generación de valor de negocio $\left(R^{2}\right)$ se puede medir / manifestar.

Tabla 11. Clasificación de valoración formas para generar valor de negocio. Algoritmo PLS. Ordenadas de mayor a menor

\begin{tabular}{lcc}
\hline \multicolumn{1}{c}{ Variable } & Valor & $\begin{array}{c}\text { Clasificación Chin (1998, p. 323); Höck } \\
\text { (2006, p. 15) }\end{array}$ \\
\hline Y7 Incrementar Ganancias & 0.781 & Substancial \\
Y2 Incrementar Productividad & 0.756 & Substancial \\
Y5 Incrementar Rentabilidad & 0.741 & Substancial \\
Y3 Reducir Costos & 0.670 & Moderada \\
Y9 Lograr el R.O.I & 0.669 & Moderada \\
Y6 Incrementar Ventas & 0.669 & Moderada \\
Y10 Incrementar Productividad de Proyectos & 0.635 & Moderada \\
Y1 Eliminar Re Trabajos & 0.599 & Moderada \\
Y8 Incrementar Mercado & 0.594 & Moderada \\
Y4 Lograr la Satisfacción del Cliente & 0.316 & Débil \\
\hline
\end{tabular}

Fuente: Elaboración propia. Corrida del SmartPLS 2.0.M3 (Ringle et al., 2005)

Como se puede observar en la Tabla 10, la variable "Lograr la satisfacción del cliente" obtiene la más baja puntuación. Este resultado discrepa de lo encontrado en la literatura consultada en la que se habla de que cumplir con esta variable es una de las principales maneras de generar valor de negocio. Lo anterior significa la necesidad de culturizar a las personas responsables de gestionar y ejecutar los proyectos de TIC sobre la importancia del tema, tal y como ya se comentó con anterioridad.

Considerando lo mencionado en párrafos anteriores y la experiencia profesional de los autores, es necesario sensibilizar al personal responsable de administrar y realizar la gestión de proyectos de tecnología de información y comunicaciones, del impacto financiero que tienen sus decisiones y manera de administrar dichos proyectos, deben ampliar su visión para integrar otros criterios con los cuáles puedan lograr satisfacer a sus clientes y entregar 
resultados de negocio. Se requiere culturizar a dicho personal de la importancia de que en su desempeño de la gestión de proyectos, traduzcan los resultados de los proyectos a resultados del negocio, con lo cual se conseguiría elevar la importancia que imprimen a la gestión de las variables mencionadas que hoy en día no lo hacen. A continuación se muestra una figura propuesta por Jack Duggal que ejemplifica gráficamente este replanteamiento de los resultados.

Figura 4. Next level up: How do you measure project success

Rethinking the triple constraint

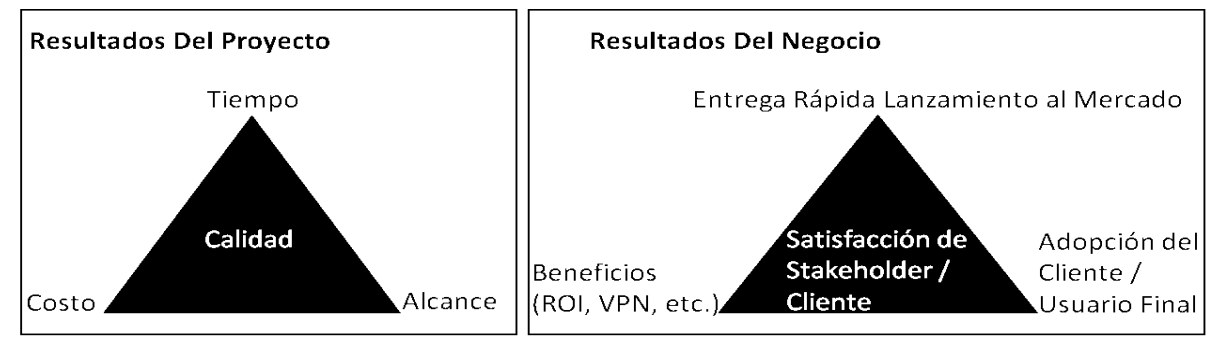

Fuente: Elaboración propia tomado de Duggal, S. J. (2010a)

\section{Referencias}

Andersen, B., Henriksen, B. \& Aarseth, W. (2007). Benchmarking of project management office establishment: Extracting best practices, Journal of Management in Engineering, 23(2), 97-104.

Baker, B. (2007). Definition impossible, PM Network, 21(6), 25.

Barclay, D., Higgins, C. \& Thompson, R. (1995). The partial least squares (PLS) approach to causal modeling: Personal computer adoption and use as an illustration, Technology Studies, Special Issue on Research Methodology, 2(2), 285-309.

Brantley, W. (2007). Justifying the value of a project management deployment in your organization through an ROI impact study. 2007 PMI Global Congress Proceedings. Atlanta, GA: PMI, 1-10.

Chin, W. W. (1998a). Issues and opinion on structural equation modeling, MIS Quarterly, 22(1), 7-16.

Chin, W. W. (1998b). The partial least squares approach to structural equation modeling. En Marcoulides, G. A. (ed.) Modern methods for business research. Mahwah, NJ: Lawrence Erlbaum Associates, Publisher, 295-336. 
Chin, W. W., Marcolin, B. L. \& Newsted, P. R. (2003). A partial least squares latent variable modeling approach for measuring interaction effects: Results from a Monte Carlo simulation study and an electronic-mail emotion, Information Systems Research, 14(2), 189-217.

Cohen, J. (1988). Statistical power analysis for the behavioral sciences. Mahwah, New Jersey: Lawrence Erlbaum.

Dai, C. X. \& Wells, W. G. (2004). An exploration of project management office features and their relationship to project performance, International Journal of Project Management, 22(7), 523-532.

Duggal, S. J. (2006). Next generation PMO. The Secret of a Successful PMO. 2006 PMI Global Congress Proceedings. Seattle: PMI.

Duggal, S. J. (2009). In the pursuit of the elusive: Showing PMO value. 2009 PMI Asia Pacific Congress Proceedings. Kuala Lumpur, Malaysia: PMI.

Duggal, S. J. (2010a). Next level up: How do you measure project success Rethinking the triple constraint. PMI Community Post.

Duggal, S. J. (2010b). To measure project managers, create a performance scorecard. PMI Global Congress 2009. Kuala Lumpur, Malaysia: PMI.

Fornell, C.; D.F. Larcker (1981). Evaluating structural equation models with unobservable variables and measurement error, Journal of Marketing Research, 18(1), 39-50.

Garson, G. D. (2016). Partial Least Squares: Regression and structural equation models. Asheboro, NC: Statistical Associates Publishers.

Geisser, S. (1974). A predictive approach to the random effects model, Biometrika, 61(1), 101-107.

Hair, J. F. Jr., Hult, G. Tomas M., Ringle, C. \& Sarstedt, M. (2014). A primer on partial least squares structural equation modeling (PLS-SEM). Thousand Oaks, CA: Sage Publications.

Hair, J. F. Jr., Anderson, R. E., Tatham, R. L. \& Black, W. C. (1999). Análisis multivariante. 5a. Edición. Madrid: Prentice Hall lberia.

Hair, J., Ringle. C. M. \& Sarstedt, M. (2011). PLS-SEM: Indeed a silver bullet, Journal of Marketing Theory and Practice, 19(2), 139-151.

Henseler, J., Ringle, C. M. \& Sinkovics, R. R. (2009). The use of Partial Least Squares path modeling in international marketing, Advances in International Marketing, 20, 277319.

Hernández, R., Fernández, C. \& Baptista, P. (2006). Metodología de la investigación. Toluca: McGraw Hill.

Hobbs, B. \& Aubry, M. (2007). A multi-phase research program investigating Project Management Offices (PMOs): The results of phase 1, Project Management Journal, 38(1), 74-86.

Höck. C, Ringle, C. M. \& Sarstedt, M. (2010). Management of multi-purpose stadiums: Importance and performance measurement of service interfaces. Journal Technology and Management, 14(2/3), 188-207.

Höck, M. \& Ringle, C. M. (2006). Strategic networks in the software industry: An empirical analysis of the value continuum. IFSAM VIIlth World Congress, Berlin 2006, 1-18. 
Hurt, M. \& Thomas, J. L. (2009). Building value through sustainable project management offices, Project Management Journal, 40(1), 55-72.

Kendall, G. I. \& Rollins, S. C. (2003). Advanced project portfolio management and the PMO. Multiplying ROI at warp speed. Boca Raton: J. Ross Publishing.

Kwak, Y. H. \& Xiao Yi Dai, Ch. (2000). Assessing the value of project management offices (PMO). PMI Research Conference 2000 Proceedings, Paris: PMI. 1-8.

Letavec, C. J. (2006). The Project Management Office. Establishing, managing and growing the value of a PMO. Boca Raton: J. Ross Publishing.

Letavec, C. J. \& Bolles, D. (2010). The PMOSIG program management office handbook. Strategic and tactical insights for improving results. Boca Raton: J. Ross Publishing.

Nunnally, J. C. (1978). Psychometric theory. New York: McGraw Hill.

O'Brochta, M. (2002). How to demonstrate the value of the PMO. Information Management. p.1, párr.1. How your business works. New York. Recuperado de: http://www.information-management.com/infodirect/2011_240/Project-managementvalue-KPI-ROI-10022347-1.html

Pinto, A., De Matheus, M. F. \& Levin, G. (2010). The PMO maturity cube, a Project Management Office maturity model. 2010 PMI Research and Education Congress 2010, Washington D.C: PMI.

PMI PMBOK Guide (2012). The Project Management Body of Knowledge (PMBOK). New Town Square: Book Store Administrator, PMI Publications.

PULSE (2012). Pulse of the profession. The impact of PMO's on the strategies implementation. Newtown Square: Project Management Institute.

PULSE (2013). Pulse of the profession - The impact of PMO's on the strategies implementation. Newtown Square: Project Management Institute

PULSE (2014). PMl's pulse of the profession: The high cost of low performance. Newtown Square: Project Management Institute.

Ringle, C., Wende, S. \& Will, A. (2005). SMARTPLS 2.0. Hamburg: Hamburg University.

Ruiz, M.; Pardo, A.; San Martín, R. (2010). Modelos de ecuaciones estructurales. Papeles del Psicólogo, 31(1), 34-45.

Stanleigh, M. (2009). Underscoring the value - and ensuring the survival - of the Project Management Office, Ivey Business Journal, 73(4), 7-12.

Stone, M. (1974). Cross-validatory choice and assessment of statistical predictions, Journal of the Royal Statistical Society, 36(2), 111-147.

Ward, J. L. (2010). The PMO in hard times: Adding value or adding cost. 2010 PMI Global Congress Proceedings. Milan: PMI. 